\title{
FitSearch: a robust way to interpret a yeast fitness profile in terms of drug's mode-of-action
}

\author{
Minho Lee ${ }^{1 \dagger}$, Sangjo Han ${ }^{2 \dagger}$, Hyeshik Chang ${ }^{3 \dagger}$, Youn-Sig Kwak ${ }^{4}$, David M Weller ${ }^{5}$, Dongsup Kim ${ }^{1 *}$ \\ From The Eleventh Asia Pacific Bioinformatics Conference (APBC 2013) \\ Vancouver, Canada. 21-24 January 2013
}

\begin{abstract}
Background: Yeast deletion-mutant collections have been successfully used to infer the mode-of-action of drugs especially by profiling chemical-genetic and genetic-genetic interactions on a genome-wide scale. Although tens of thousands of those profiles are publicly available, a lack of an accurate method for mining such data has been a major bottleneck for more widespread use of these useful resources.

Results: For general usage of those public resources, we designed FitRankDB as a general repository of fitness profiles, and developed a new search algorithm, FitSearch, for identifying the profiles that have a high similarity score with statistical significance for a given fitness profile. We demonstrated that our new repository and algorithm are highly beneficial to researchers who attempting to make hypotheses based on unknown modes-ofaction of bioactive compounds, regardless of the types of experiments that have been performed using yeast deletion-mutant collection in various types of different measurement platforms, especially non-chip-based platforms.

Conclusions: We showed that our new database and algorithm are useful when attempting to construct a hypothesis regarding the unknown function of a bioactive compound through small-scale experiments with a yeast deletion collection in a platform independent manner. The FitRankDB and FitSearch enhance the ease of searching public yeast fitness profiles and obtaining insights into unknown mechanisms of action of drugs.

FitSearch is freely available at http://fitsearch.kaist.ac.kr.
\end{abstract}

\section{Background}

The collection of yeast deletion strains has been a powerful tool for systematic genome-wide functional analysis. A budding yeast deletion-mutant library has been available for more than ten years [1], and recently a fission yeast collection has also become available [2]. In particular, strain-specific molecular barcodes allow quantitative functional profiling of pooled deletion strains by using a TAG oligonucleotide microarray [3]. Among many types of functional profiles, the chemical-genetic profiles express quantitative values of growth defects of deletion strains in the presence of certain chemicals. The compendium of

\footnotetext{
*Correspondence: kds@kaist.ac.kr

† Contributed equally

'Department of Bio and Brain Engineering, Korea Advanced Institute of Science and Technology, 291 Daehak-ro, Yuseong-Gu, Daejeon, 305-701, Republic of Korea

Full list of author information is available at the end of the article
}

chemical-genetic profiles of heterozygous and homozygous deletion strains for a large number of chemicals has been successfully used to identify direct target proteins of drugs $[4,5]$ as well as exploring their modes-of-action $[6,7]$. Such profile data can also be a valuable resource for many other applications in chemical genomics. In S. cerevisiae, thousands of chemical-genetic profiles have been generated so far and are publicly available [8]. Fitness data have been deposited in a recently developed public database called fitDB [8]; this database, however, only provides limited tools such as an online-interface for searching with keywords such as yeast ORFs or drug names.

In a large-scale study using a single measurement platform, researchers can easily compare fitness profiles using several well-known similarity measures. Then, by clustering the profiles, they can group bioactive compounds with a similar mode-of-action and make a plausible hypothesis 
about the unknown mode-of-action of a drug [7]. However, chemical-genetic profiles can be generated using many different measurement platforms such as DNA chip-based parallel measurements [4,5,7,9], high-density colony measurement on agar plates [10], high-density well plate-based optical density [11] or fluorescence [12] measurements in liquid culture. Moreover, the fitness scores can be expressed in many different ways (i.e., fold-ratio, zscore, p-value, ranks, or binary values expressing growth defects). Such difficulties complicate the process of relating one profile to another. This problem becomes more severe when only a limited number of yeast fitness profiles for a drug of interest are available, and more so when such profiles have been generated by non-chip-based measurement platforms. In such cases, it is very difficult to perform data-mining against the tens of thousands of public fitness profiles that may contain valuable information on the mode-of-action of the drug of interest.

In this study, we developed FitRankDB as a general repository of fitness profiles, and FitSearch as a new fitness similarity search algorithm which compares fitness profiles and calculates their similarity score and the corresponding statistical significance, regardless of the types of experimental setup by which they have been generated (Figure 1). The FitRankDB and FitSearch web service provides an uncomplicated means for searching tens of thousands of public yeast fitness profiles and obtaining insight into unknown modes-of-action of drugs.

\section{Methods}

The ultimate objective of FitSearch is to provide a computational tool for interpreting any type of yeast fitness profiles in terms of the mode-of-action of a drug through comparison of various types of yeast fitness profiles and publicly available functional annotations. To do this, we first needed to create a general repository of yeast fitness profiles combined with genetic interaction information. Next, and more importantly, we needed a universal similarity measure for comparing profiles in a biologically meaningful way. Finally, for efficient and wider use of the resources, it was necessary to develop an easy-to-use public server in which users can choose and run several web applications, depending on their specific needs.

\section{FitRankDB, a general repository of yeast fitness profiles}

Genome-wide yeast fitness profiles, using a yeast deletion library, have been used to infer the mode-of-action of a drug, genetic interactions, such as synthetic lethality, and functional annotation of unknown genes. We categorized those fitness profiles according to a yeast deletion library and a treatment, and collected them from public resources (Table 1). To make our collection a general repository for yeast fitness profiles, we defined a flexible standard file format using YAML (YAML Ain't Markup Language) to express all relevant information available. This yeast fitness YAML (called FitYAML) is a very simple format, which consists of three-letter keywords and their corresponding values. Currently, 32 three-letter keywords are defined, but this definition is scalable (see keyword definitions and download FitYAML at the website). Our internal curation system automatically extracts ranks of raw fitness scores and experimental information from FitYAML, and stores them into FitRankDB. For efficient computation, FitRankDB has two types of databases, Berkeley DB for rank information, and MySQL for experimental information. In addition, we collected various functional module definitions, such as protein complexes, to assist biological interpretation of fitness profiles into FitRankDB.

Biogrid is a manually curated database, which provides various types of genetic interactions. It also contains information that enables us to distinguish query genes from array genes in a genetic interaction assay. To construct genetic-genetic profiles from the biogrid genetic interaction data, we extracted only the synthetic lethality (SL), synthetic growth defect (SD) and phenotypic enhancement (PE) datasets in S. cerevisiae, and ranked the array genes corresponding to each query gene according to the degree of growth defect: $\mathrm{SL}>\mathrm{SD}>\mathrm{PE}$. In this procedure, array genes against the same query gene reported in different papers are merged (named 'Biogrid merged') or are separately deposited (named 'Biogrid individual') in FitRankDB. In addition, array genes against a query gene are assumed to be genes of strains with significant growth defects determined by genome-scale screening, and the query gene considered to be identified from a genetic treatment, such as gene knockout. Other large genetic interaction datasets from Epistatic Mini Array Profiles (E-MAP), were downloaded at http://interactome-cmp.ucsf.edu and deposited into FitRankDB in a similar way: 'chromosome function E-MAP' [13], 'signaling E-MAP' [14], 'early secretory pathway E-MAP' [15] and 'RNA processing E-MAP' [16].

\section{FitSearch, a rank cutoff optimizer as a search engine}

Suppose that we are investigating whether two chemicals share a similar mode-of-action. One way to do this is to measure the similarity between the two fitness profiles of chemicals that may have been generated from different measurement platforms. Then, a requisite property for a new similarity measure is that the more similar the modes-of-action of the two chemicals are, the greater the similarity score should be. Realizing that the main difficulty in developing a biologically meaningful similarity measure arises from the fact that the profiles may have been generated from different types of experimental setup and that their fitness values may have been expressed in different ways, we first transformed the fitness values of 


\section{Target}

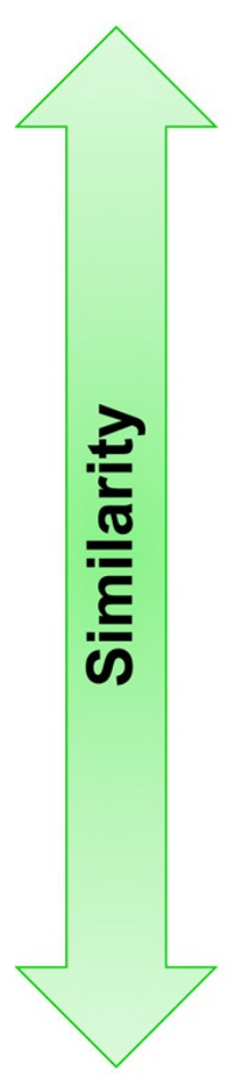

Query

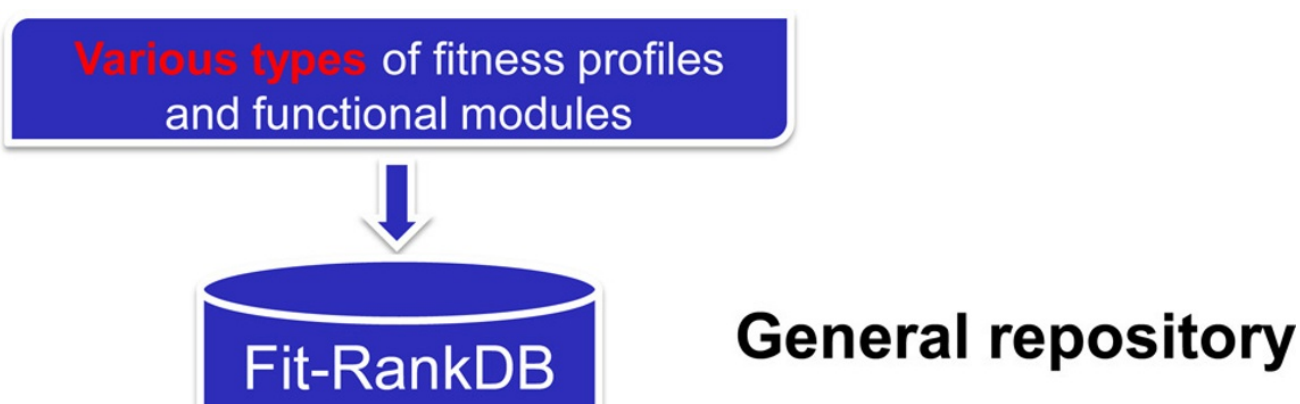

$\mathbf{1}$

\section{Rank-cutoff optimizer}

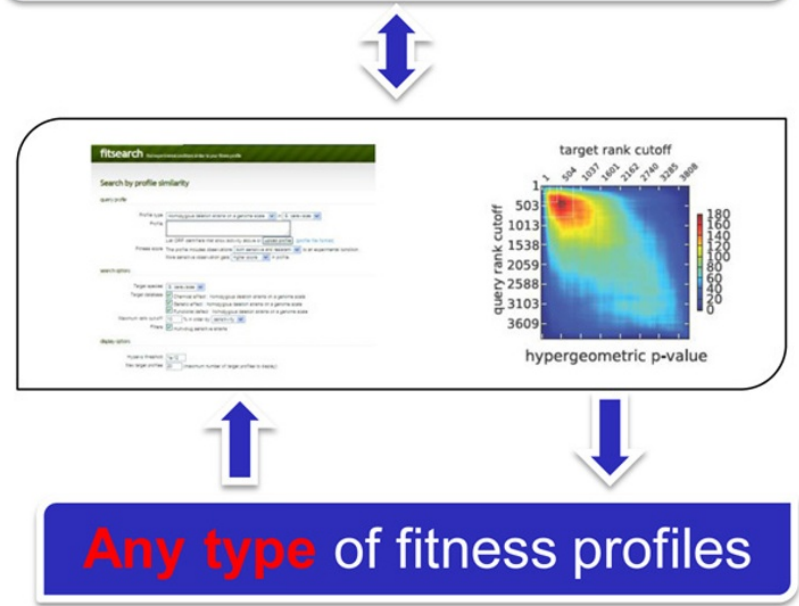

Search engine

Web interface

Figure 1 Overall scheme of FitSearch. Although researchers have only one or two yeast fitness profiles to their drugs of interest that have unknown toxicity mechanisms, they can easily perform data-mining against tens of thousands of public fitness profiles in order to obtain insight into the mechanism through the FitSearch website (http://fitsearch.kaist.ac.kr). When any type of yeast fitness profile is submitted as a query in the website, a similarity search to other public resources is performed by rank-cutoff optimizer through the FitSearch engine, which is a newly developed method using rank-based overlapping statistics (see the details in the Methods). Since available public resources are deposited in FitRankDB as a general repository for the FitSearch engine (see the details in the Methods), the similarity search can be performed more efficiently, thoroughly, and rapidly in the FitSearch website. Finally, users scrutinize characteristics of a list of drugs similar to their drug of interest and obtain clues or plausible hypotheses, which could also help them to design further bioassays.

each strain into their ranks. Next, considering that only the highly ranked strains that are significantly affected by a given chemical treatment are informative for inferring its mode-of-action, we chose the rank cutoff values for the two fitness profiles. Unlike previous methods where cutoff values were chosen rather arbitrarily, we developed an

Table 1 Different types of yeast fitness profiles deposited in FitRankDB.

\begin{tabular}{|c|c|c|c|}
\hline Type of treatment & Type of genome-wide deletion library & Type of fitness profile & Profile \# \\
\hline Chemical effect $^{1}$ & Homozygous deletion strains ${ }^{3}$ & Chemical-genetic (Hom) & 918 \\
\hline Chemical effect & Heterozygous deletion strains ${ }^{3}$ & Chemical-genetic (Het) & 1,530 \\
\hline Genetic effect ${ }^{2}$ & Homozygous deletion strains & Genetic-genetic (Hom) & 12,419 \\
\hline
\end{tabular}

See the details at http://pombe.kaist.ac.kr/fitsearch/statistics/

${ }^{1}$ For example, drug, bioactive compounds or natural crude extracts

${ }^{2}$ For example, knock-out, over-expression or mutation of a gene

${ }^{3}$ Homozygous (or haploid) and heterozygous (or diploid) deletion collections of S. cerevisiae and S. pombe are commercially available at Open Biosystems (http:// www.openbiosystems.com) and Bioneer (http://pombe.bioneer.co.kr), respectively. 
efficient dynamic programming algorithm to choose the two optimal rank cutoff values.

This algorithm named "rank cutoff optimizer" finds the optimal rank cutoffs by maximizing the statistical significance for overlap of the same set of strains in the two lists. The probability of overlapping by chance is known to follow the hyper-geometric distribution [17]. Suppose that there are $m_{i j}$ strains that co-occur in both the query profile, with the rank cutoff $i$, having $q_{i}$ strains and the target profile, with the rank cutoff $j$, having $t_{j}$ strains. Then the probability of such co-occurrence happening by random chance, $p\left(m_{i j}\right)$, is given by

$$
p\left(m_{i j}\right)=\frac{C\left(q_{i}, m_{i j}\right) C\left(n-q_{i}, t_{j}-m_{i j}\right)}{C\left(n, t_{j}\right)}
$$

where $n$ is the total number of strains, and $C(n, m)$ is the number of ways of choosing $m$ strains out of $n$ strains. Of particular interest is the cumulative probability of $p(m)$,

$$
H p\left(m_{i j}, q_{i}, t_{j} ; n\right)=\sum_{m=m_{i j}}^{\min \left(q_{i}, t_{j}\right)} p(m)
$$

which is simply the $p$-value. In addition, a p-value considering multiple hypothesis correction is defined using Bonferroni correction, which is a stringent correction method, given by

$$
\tilde{p}=N p
$$

where $N$ is represents the number of tests. This correction eventually increases the p-value. The rank cutoff optimizer calculates the optimal rank cutoffs, $i *$ and $j *$, by minimizing the $\mathrm{p}$-value, i.e.,

$$
\left(i^{*}, j^{*}\right)=\underset{i, j=1 . . k}{\arg \min } H p\left(m_{i j}, q_{i}, t_{j} ; n\right)
$$

and reports both the overlapping significance defined as $-\log _{10}(p$-value $)$ and the overlapping score, which is the Tanimoto coefficient given by

$$
T=\frac{m_{i^{*} j^{*}}}{q_{i^{*}}+t_{j^{*}}-m_{i^{*} j^{*}}}
$$

We will explain the rank cutoff optimizer step by step with a toy example. Suppose that we have two fitness profiles, one as a query and the other as a target. The first step is to convert the fitness values of each strain into their ranks as shown in Figure 2A. After each rank in the query and the target is sorted by their corresponding strain names as shown in Figure 2A, the rank matches of each strain can be expressed as a "match matrix" (M) as shown in the upper panel of Figure 2B. In the $\mathbf{M}$, the rows represent the ranks in the query, and columns represent the ranks in the target, and the value indicates the strain number with the same rank in both the query and the target.

Step 2. Constructing an accumulated match matrix (A): Efficient calculation of the match number accumulated under all possible rank-cutoffs of the query and the target can be achieved by dynamic programming as follows:

$$
A_{i j}=M_{i j}+A_{i j-1}+A_{i-1 j}-A_{i-1 j-1}
$$

In the equation (6), $M_{i j}$ is the match number in the rank $i$ of the query and the rank $j$ of the target, and $A_{i j}$ is the match number accumulated under the rank-cutoff $i$ of the query and the rank-cutoff $j$ of the target, which is schematically depicted in Figure 2B. This accumulated match matrix (A) provides the objective function, $\mathrm{Hp}$, with all possible parameters for optimization. In $\mathbf{A}$, values represent $m_{i j}$ as the overlapped strain number in the equation (2), its row-names $q_{i}$ indicate the query strain number and column-names $t_{j}$ indicate the target strain number in their respective rank-cutoffs $i$ and $j$, and the column or row length $n$ represents the size of the population. In the toy example of Figure 2, the maximal rank-cutoff $k$ is set to the same as $n$.

Step 3. Finding the optimal rank-cutoffs from a cumulative hyper-geometric $p$-value matrix $(\mathrm{P})$ : The cumulative hyper-geometric $p$-values are calculated through the equation (2) of the objective function, $H p$ for all possible rank-cutoffs, stored in the $\mathbf{P}$, and then used to find the rank-cutoffs with the minimized $p$ value as described in the equation (1). In the toy example of Figure 2, the query rank-cutoff of 5 and the target rank-cutoff of 5 show the minimal $p$-value, 0.004 (Figure $2 \mathrm{C})$. This means that the best significant relationship between the query and the target in terms of overlap is observed at those rank-cutoffs, which are termed optimal rank-cutoffs in our study.

After this optimization, we can define the similarity between the query and the target at their optimal rankcutoffs. In our study, we use two values: one is the minimized $p$-value as an overlapping significance, and the other is the Tanimoto coefficient as an overlapping score.

\section{Web application as the user interface}

To search the similarities with all the profiles deposited in the FitRankDB using the rank-cutoff optimizer against a query profile, we provide users with two web frontends (Table 2). This web application uses a pythonbased Django framework and the rank-cutoff optimizer as the third party program implemented by $\mathrm{C}$ program. Significant top search results can be differently interpreted according to the types of query and target. Some clear interpretations are shown in Table 3. 
A

\begin{tabular}{|l|c|c|c|c|c|c|c|c|c|c|}
\hline Strain & a & b & c & d & e & f & g & h & i & j \\
\hline Query rank & 1 & 2 & 3 & 4 & 5 & 6 & 8 & 7 & 10 & 9 \\
\hline Strain rank & 2 & 3 & 1 & 5 & 4 & 7 & 10 & 8 & 9 & 6 \\
\hline
\end{tabular}

B

\begin{tabular}{|c|c|c|c|c|c|c|c|c|c|c|c|}
\hline M & & 1 & 2 & 3 & 4 & 5 & 6 & 7 & 8 & 9 & 10 \\
\hline 1 & & & 1 & & & & & & & & \\
\hline 2 & & & & 1 & & & & & & & \\
\hline 3 & & 1 & & & & & & & & & \\
\hline 4 & & & & & & 1 & & & & & \\
\hline 5 & & & & & 1 & & & & & & \\
\hline 6 & & & & & & & & 1 & & & \\
\hline 7 & & & & & & & & & 1 & & \\
\hline 8 & & & & & & & & & & & 1 \\
\hline 9 & & & & & & & 1 & & & & \\
\hline 10 & & & & & & & & & & 1 & \\
\hline A & 0 & 1 & 2 & 3 & 4 & 5 & 6 & 7 & 8 & 9 & 10 \\
\hline$\theta$ & $\theta$ & $\theta$ & $\theta$ & $\theta$ & $\theta$ & $\theta$ & $\theta$ & $\theta$ & $\theta$ & $\theta$ & $\theta$ \\
\hline 1 & 0 & 0 & 1 & 1 & 1 & 1 & 1 & 1 & 1 & 1 & 1 \\
\hline 2 & 0 & 0 & 1 & 2 & 2 & 2 & 2 & 2 & 2 & 2 & 2 \\
\hline 3 & 0 & 1 & 2 & 3 & 3 & 3 & 3 & 3 & 3 & 3 & 3 \\
\hline 4 & 0 & 1 & 2 & 3 & 3 & $\rightarrow 4$ & 4 & 4 & 4 & 4 & 4 \\
\hline 5 & 0 & 1 & 2 & 3 & 4 & 5 & 5 & 5 & 5 & 5 & 5 \\
\hline 6 & 0 & 1 & 2 & 3 & 4 & 5 & 5 & 6 & 6 & 6 & 6 \\
\hline 7 & $\theta$ & 1 & 2 & 3 & 4 & 5 & 5 & 6 & 7 & 7 & 7 \\
\hline 8 & 0 & 1 & 2 & 3 & 4 & 5 & 5 & 6 & 7 & 7 & 8 \\
\hline 9 & 0 & 1 & 2 & 3 & 4 & 5 & 6 & 7 & 8 & 8 & 9 \\
\hline 10 & 0 & 1 & 2 & 3 & 4 & 5 & 6 & 7 & 8 & 9 & 10 \\
\hline
\end{tabular}

C

\begin{tabular}{|c|c|c|c|c|c|c|c|c|c|c|}
\hline $\mathbf{P}$ & 1 & 2 & 3 & 4 & 5 & 6 & 7 & 8 & 9 & 10 \\
\hline 1 & 1.000 & 0.200 & 0.300 & 0.400 & 0.5 do & 0.600 & 0.700 & 0.890 & 0.900 & 1.000 \\
\hline 2 & 1.000 & 0.378 & 0.067 & 0.133 & 0.222 & 0.333 & 0.467 & 0.622 & 0.800 & 1.000 \\
\hline 3 & 0.300 & 0.067 & 0.008 & 0.033 & 0.083 & 0.167 & 0.292 & 0.467 & 0.700 & 1.000 \\
\hline 4 & 0.400 & 0.133 & 0.033 & 0.119 & 0.024 & 0.071 & 0.167 & 0.333 & 0.600 & 1.000 \\
\hline 5 & 0.500 & 0.222 & 0.083 & 0.024 & 0.004 & 0.024 & 0.083 & 0.222 & 0.500 & 1.000 \\
\hline 6 & 0.600 & 0.333 & 0.167 & 0.071 & 0.024 & 0.119 & 0.033 & 0.133 & 0.400 & 1.000 \\
\hline 7 & 0.700 & 0.467 & 0.292 & 0.167 & 0.083 & 0.333 & 0.183 & 0.067 & 0.300 & 1.000 \\
\hline 8 & 0.800 & 0.622 & 0.467 & 0.333 & 0.222 & 0.667 & 0.533 & 0.378 & 1.000 & 1.000 \\
\hline 9 & 0.900 & 0.800 & 0.700 & 0.600 & 0.500 & 0.400 & 0.300 & 0.200 & 1.000 & 1.000 \\
\hline 10 & 1.000 & 1.000 & 1.000 & 1.000 & 1.000 & 1.000 & 1.000 & 1.000 & 1.000 & 1.000 \\
\hline
\end{tabular}

Figure 2 Toy example showing how the rank-cutoff optimizer works. (A) Ranks of each strain in virtual two query and target yeast fitness profiles to be compared are supposed to be deposited in Fit-RankDB. These profiles are also supposed to be generated using a virtual yeast deletion library comprising strain a to j. (B) Efficient calculation of a match number (or an overlapped strain number) accumulated under all possible rank-cutoffs of the query and the target by Dynamic programming (see the details in the Methods). For this calculation, first, rank matches of each strain should be expressed as the match matrix (M). In the M matrix, its row represents 'ranks in the query', its column 'ranks in the target', and its value 'the strain number with same rank in the query and the target'. Then, the current accumulated match number (in redcolored cell in the A matrix) is calculated by adding the current match number (in the orange-colored cell in the $\mathrm{M}$ matrix) to the previous accumulated match number (sky-colored cell plus purple-colored cell minus gray-colored cell in the A matrix). In this way, the accumulated match numbers regarding to all possible rank-cutoffs are efficiently calculated and stored in the A matrix. (C) The matrix of cumulative hypergeometric $p$-values $(P)$ is filled by calculating the equation (2) as the objective function ( $\mathrm{Hp}$ ) regarding to all possible rank-cutoffs, and used to find the rank-cutoffs with the minimized p-value as described in the equation (3), called optimal rank-cutoffs. The A matrix provides all of the parameters needed for equations (2) and (3) as follows: Its values represent the overlapped strain number in the equation (2); its row-names, the query strain number; its column-names, the target strain number in their respective rank-cutoffs; and its column or row length, the size of population. When the maximal rank-cutoff is set to 10 in the toy example, the query rank-cutoff 5 and the target rank-cutoff 5 shows the minimal $p$-value, 0.004. At those optimal rank-cutoffs, overlapping significance (hyper-geometric $p$-value) and overlapping score (Tanimoto coefficients) can be expressed as the similarity between the query and the target. 
Table 2 Available frontends in FitSearch web site.

\begin{tabular}{ll}
\hline Option & Description \\
\hline FitSearchp & Search pre-compiled fitness rank database (FitRankDB) with a fitness profile of user. \\
FitSearchd & Search FitRankDB with the profile specified in FitRankDB. \\
\hline
\end{tabular}

There are more details in 'help' page in the web site.

\section{Results}

In FitSearch, we consider the following two design principles, universality and simplicity (See details in Methods). In brief, we adopt the use of rank statistics to compare two fitness profiles. This implies that any type of fitness scores can be universally converted into their corresponding ranks. The similarity of the two rank-transformed profiles can be easily calculated by rank-based comparison methods if we consider all of the profiles. In most situations, however, except for a relatively small number of top-ranked strains that are severely affected by the given drug, most strains can be considered as producing noise signals. Therefore, removal of such non-informative strains is necessary in order to calculate more accurate similarity measures and to make more meaningful comparison between profiles that may have been generated from different experimental treatments. An important question then arises: how should we define informative or non-informative strains? In other words, how should we set the optimal rank cutoffs for the two given profiles? The rank cutoff is an arbitrary value, can vary depending on viewpoint of the researcher, and is difficult to choose in advance for a pair of profiles.

We solved this problem using an optimization method. Among all possible combinations of rank-cutoff values for a pair of profiles, FitSearch finds the optimal rank cutoffs by minimizing the $\mathrm{p}$-value for the co-occurrence of the same set of strains in the two lists by chance, and finally returns two types of scores at those optimal rank cutoffs, viz., the Tanimoto coefficient and the p-value (Figure 2). These two scores based on overlapping strains efficiently display the similarity between profiles (Figure 3). Researchers do not need to define the cutoff values, which are automatically calculated by an internal optimizer in FitSearch.

To investigate whether FitSearch correctly identified fitness profiles sharing a similar mode-of-action, we performed a series of case studies, and validated the results by using prior knowledge and by performing experiments.

\section{Case study 1: comparing chemical-genetic profiles from different measurement platforms}

Several high-throughput fitness profile datasets generated by using different platforms were deposited in FitRankDB. For example, the compendium named 'Cell, 2004' [4] contains heterozygous fitness profiles from the Merck company and fitDB collection [8], and 'Cell, 2006' contains homozygous fitness profiles from the Boone group [7]. In addition, the compendium named 'Science, 2008' [8] contains both homozygous and heterozygous fitness profiles from the fitDB collection. In this case study, we tested whether our method successfully identified the correct relationships between the chemicalgenetic profiles generated from different measurement platforms.

Clustering the drugs according to the similarity between their corresponding chemical-genetic profiles provides insights into the unknown modes-of-action of drugs. This has been well demonstrated in 'Cell, 2006' where fitness profile data were generated from the platform using genome-wide homozygous deletion strains (i.e. a haploid collection). To generalize such an approach, we developed a method for measuring the similarity between chemicalgenetic profiles generated by various types of measurement platforms (see Methods for details). We tested our new method using the chemical-genetic profiles of clotrimazole, latrunculin B, beomyl and cisplatin in 'Cell, 2006' as a query, and searched the combined database of 'Science, 2008' and 'Cell, 2006' which contain roughly 1,000 profiles. Under appropriate two-way cutoffs of overlapping score and significance, the top-ranked target profiles were either fitness profiles of the query drugs from

Table 3 Biological interpretation about similarity between two fitness profiles

\begin{tabular}{|c|c|c|}
\hline Query fitness profile & Target fitness profile & Biological interpretation of similar target treatment \\
\hline Chemical-genetic (Hom) & $\begin{array}{l}\text { Chemical-genetic } \\
\text { (Hom) }\end{array}$ & Chemical effect (i.e. drug toxicity) with similar mode-of-action \\
\hline Chemical-genetic (Het) & Chemical-genetic (Het) & $\begin{array}{l}\text { Chemical effect with similar mode-of-action; Finding common direct drug target } \\
\text { protein }\end{array}$ \\
\hline Chemical-genetic (Hom) & Genetic-genetic (Hom) & Genetic effect (i.e. knock-out and mutations) on direct drug target protein gene \\
\hline $\begin{array}{l}\text { Chemical/genetic-genetic (Hom/ } \\
\text { Het) }\end{array}$ & $\begin{array}{l}\text { Biological } \\
\text { functional annotation: } \\
\text {-Gene ontology } \\
\text {-Protein complexes }\end{array}$ & Biological functions related to chemical or genetic effect \\
\hline
\end{tabular}



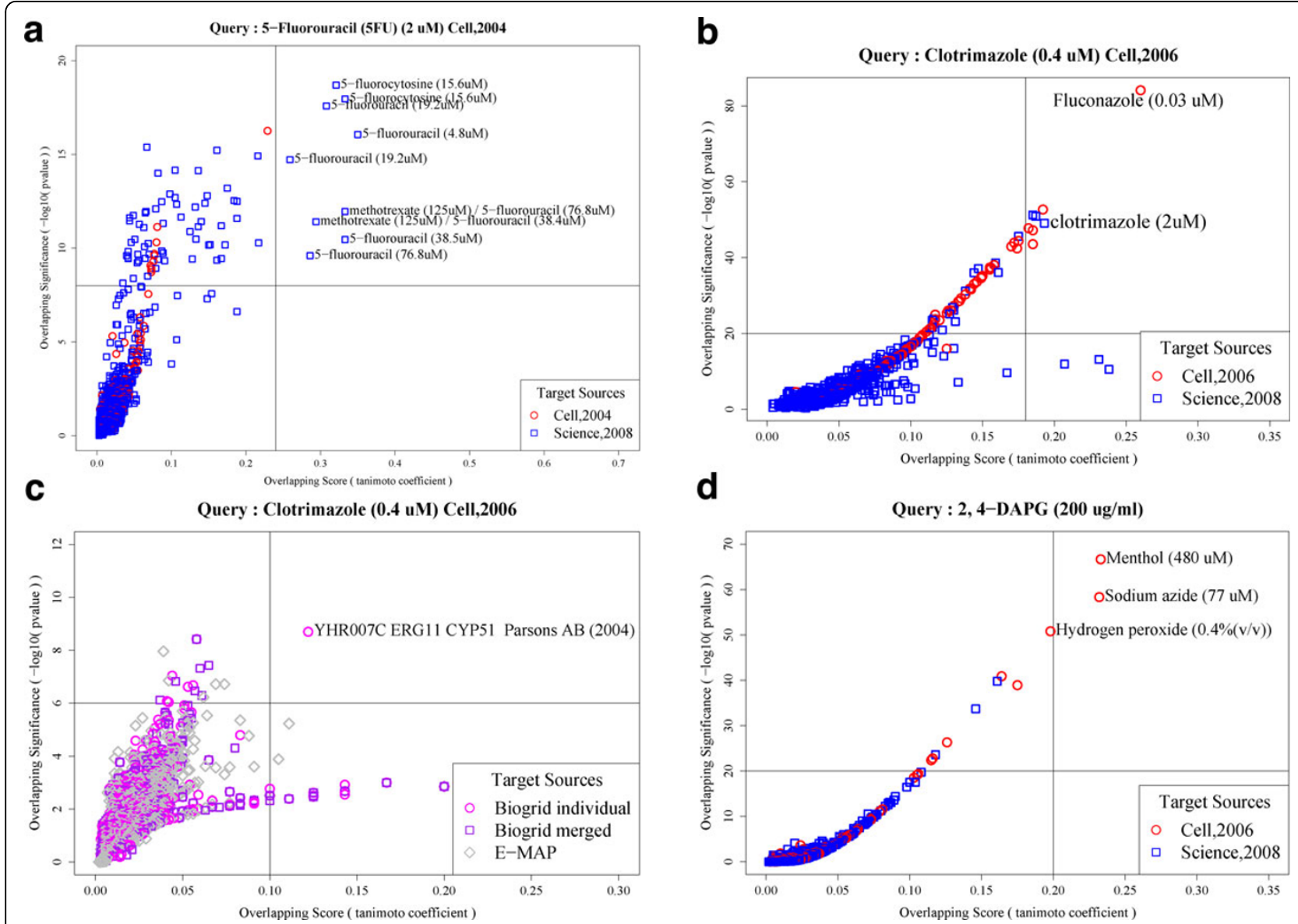

Figure 3 Plot of an overlapping score and an overlapping significance as two-way cutoffs to show the most similar chemical or genetic effects to a query's effect. (A) Two-way cutoff plot of the most similar chemical effects to the 5-Fluorouracil's effect. (B) Two-way cutoff plot of similar chemical effects to clotrimazole's effect. (C) Two-way cutoff plot of the most similar genetic effects to clotrimazole's effect. (D) Two-way cutoff plot of the most similar chemical effects to DAPG's effect. Target sources mean public chemical-genetic or genetic-genetic yeast profiles.

different platforms or those of chemicals known to have a common mode-of-action in common with the query (Additional file 1). For example, the best hits for clotrimazole $(0.4 \mu \mathrm{M})$ in 'Cell, 2006' are clotrimazole with a different dose $(2 \mu \mathrm{M})$ in 'Science, 2008' and fluconazole (0.03 $\mu \mathrm{M})$ in 'Cell, 2006' (Figure 3B). In addition, most of the target profiles of azoles from different platforms are listed in the top ranks.

In the case of genome-wide heterozygous deletion strains (i.e. a diploid collection), the main application of the chemical-genetic profiles is to find direct drug target protein candidates by screening drug-induced haploinsufficient strains $[4,5]$. Grouping of similar chemical-genetic profiles is not a typical approach, but was introduced to discover common targets and associated cellular functions for multiple classes of drugs $[5,18]$. To investigate the potential of FitSearch in a diploid collection, we selected as a query the yeast fitness profile of 5-flourouracil (5-FU), one of chemical-genetic profiles reported in 'Cell, 2004' which was the first compendium of chemical-genetic profiles based on a diploid collection. Even though this compendium was generated using roughly half of the genome-wide deletion strains, this study demonstrated that it was possible to identify the direct target protein of the drug and reported a novel target of 5-FU. We searched FitRankDB with 5-FU as a query using the FitSearchd program (Table 2). As expected, fitness profiles of 5-FU with different doses and generated from different platforms resided in the top ranks (Figure 3A). Additionally, similar chemicals, such as 5-fluorodeoxyuridine and 5-fluorocytosine showed high similarities. A synergistic effect of methotrexate and 5 -fluorouracil was also detected by the similarity search.

\section{Case study 2: comparing chemical-genetic profiles and genetic-genetic profiles}

In principle, deletion of a gene that encodes the target of an inhibitory compound should cause cellular effects 
that are similar to the inhibition of the target by drug treatment. In a proof-of-principle experiment, it was shown that for five different chemicals the chemicalgenetic profiles were highly similar to the genetic interaction profiles of the target gene or genes in the target pathway [19]. To generalize this approach, we created a comprehensive compendium of global genetic interaction profiles by combining the interaction data from Biogrid [20] and E-MAP. Biogrid was deposited into FitRankDB in the forms of 'Biogrid individual' and 'Biogrid merged' (see Method for details).

For testing, we used the four chemical-genetic profiles of clotrimazole, latrunculin $\mathrm{B}$, beomyl and cisplatin in 'Cell, 2006' as a query, and searched 'Biogrid individual', 'Biogrid merged' and 'E-MAP' to find the target profiles. We found that the top-ranked target profiles were relevant to the known mode-of-action of the query chemicals (Additional file 1). In particular, the target profile of ERG11 was detected at the first rank (Figure 3C); ERG 11 is known to be a direct target protein of clotrimazole.

\section{Case study 3: experimental validation}

FitSearch is most beneficial to researchers who have performed a small-scale experiment using a yeast deletion collection. In a small-scale experiment, researchers usually generate one or two chemical-genetic profiles by colonyor OD-based platforms. That makes it difficult to perform data mining from public resources that were typically generated by chip-based platforms. To investigate how useful FitSearch is for a small-scale experiment, it was applied to obtain a hypothesis on the toxicity and mechanism of action of 2,4-diacetylphloroglucinol (2,4-DAPG). 2,4DAPG is an antibiotic produced by Pseudomonas fluorescens that plays a key role in the ability of the bacterium to suppress phytopathogenic fungi. 2,4-DAPG has broad antibiotic activity, affecting organisms ranging from bacteria to higher plants. The biosynthesis and regulation of 2,4-DAPG in P. fluorescens has been well described. However, the mode of action of the antibiotic against target fungi has not been described in detail.

For testing, we selected common mutants in a haploid collection that showed significant growth defects in colony- and OD-based screening when exposed to DAPG (manuscript in preparation), and transformed them into binary query profiles. Public chemical-genetic profiles from 'Cell, 2006' and 'Science, 2008' were used as the target sources for a similar mode-of-action drug search and public genetic-interaction datasets of 'Biogrid individual', 'Biogrid merged', and 'E-MAP' were used as the target sources to find the direct drug target candidates. Under appropriate two-way cutoffs of overlapping score and significance, (1R, 2S, 5R)-2-isopropyl-5-methylcyclohexanol (menthol), sodium azide, and hydrogen peroxide were found to have similar toxicity and mechanism of action to
2,4-DAPG (Figure 3D and Additional file 1). However, no genetic effect similar to 2,4-DAPG chemical effect was detected.

\section{Predicting gene-gene interactions}

A genetic interaction can be defined as a synergetic phenotype that cannot be elucidated by simply combining the phenotypes of distinct gene perturbations. A typical way to detect genetic interaction is to make use of synthetic lethal genetic screens and synthetic dosage lethal screens. However, due to the vast number of gene-gene combinations, genome-wide screening had not been possible until the Synthetic Genetic Array (SGA) method [21] became available. Recently, genome-scale SGA analysis was used to chart the genetic interaction map in budding yeast [22]. The profiles of quantitative scores of genes in SGA screen were stored in FitRankDB. In addition, we tested whether our FitSearch algorithm could reveal genetic interactions. We carried out FitSearchd (Table 2) for all genetic fitness profiles in FitRankDB first and predicted genetic interactions if the two genetic profiles had a significant $\mathrm{p}$-value and Tanimoto coefficient ( $\mathrm{p}$-value $<1 \mathrm{e}-10$, Tc $>0.1$ ). About $12 \%$ of interactions predicted in this study are real genetic interactions according to the work by Costanzo et al. [22]. All other interactions would be either false positives or novel genetic interactions not previously detected. In Table 4, we compiled the top-10 list of these interactions sorted by Tanimoto coefficient. Although the results we obtained are not included in previous SGA screening work, there is clear evidence supporting that these predicted interactions are not false positives. These results indicate that FitSearch can find new genetic interactions, which cannot be identified by SGA analysis and that these two techniques can complement each other.

\section{Discussion}

Yeast deletion collections have been successfully used to infer modes-of-actions of drugs especially by profiling chip-based chemical-genetic and genetic-genetic interactions on a genome-wide scale $[4,5,7,9]$. In addition, tens of thousands of those profiles are publicly available. However, if researchers only have a few yeast profiles to their drugs of interest with unknown toxicity mechanisms, it is not easy to compare them with public resources to investigate whether similar profiles exist. One of the main reasons is that, typically, the chemical-genetic profiles have been generated on non-chip based measurement platforms, such as a simple 96-well spotting assay or high-density colony measurement on an agar plate [10], high-density well plate-based optical density [11], or fluorescence [12] measurements in a liquid culture. Furthermore, different fitness scoring methods are also problematic. Therefore, there is an urgent need for developing platform- and 
Table 4 FitSearch can detect genetic interactions that cannot be detected by SGA analysis.

\begin{tabular}{cccccc}
\hline Rank & Gene1 & Gene2 & Tc & P-value & Note \\
\hline 1 & YPL022W RAD1 & YML095C RAD10 & 1 & $3.44 E-29$ & Single-stranded DNA endonucleases (with each other) \\
2 & YDL040C NAT1 & YHR013C ARD1 & 0.94 & $1.18 E-317$ & Subunit of the N-terminal acetyltransferase NatA (Nat1p, Ard1p, Nat5p) \\
3 & YCR009C RVS161 & YDR388W RVS167 & 0.91 & $3.37 E-114$ & Manually curated by [27] \\
4 & YPL020C ULP1 & YKR082W NUP133 & 0.91 & $3.79 E-28$ & Overexpression of ULP1 rescues a nup133 rad27 or nup60 rad27 double \\
mutant [28]
\end{tabular}

The table shows the top 10 results that are not included in the genetic interaction list by SGA analysis [22]. Notes without references are retrieved from the Saccharomyces Genome Database (SGD) [26]. P-values, here, are corrected considering multiple tests.

scoring method-independent ways to compare fitness profiles for more efficient utilization of the public resources.

In previous studies, the conventional similarity measures, such as the Pearson correlation coefficient, were typically used for comparing fitness profiles. However, such simple similarity measures can be applied only to the profiles generated by well-controlled experiments using a single measurement platform. The probability of chance overlap by chance between two profiles was also used to measure their similarity, but was only applicable to fitness profiles with pre-defined significant thresholds [19]. Such thresholds are typically arbitrary and vary depending on the experimental setup and the researcher's viewpoint. This requires a more universal and simple way to measure similarities.

Here, we demonstrated the general usability of FitSearch as a new similarity measure of yeast fitness profiles by literature-based and experimental case studies. In the first literature-based case study, we tested whether our new method was able to find drugs with similar modes-ofaction even if their fitness profiles had been generated by different measurement platforms. A previous study showed that similarities of chemical-genetic profiles generated from the same platform tended to imply similar modes-ofaction of drugs [7]. Our new similarity measure, FitSearch, can generalize such an approach even with chemicalgenetic profiles obtained from different experimental platforms. For example, we showed that drugs very similar or the same as 5-FU and clotrimazole were detected in the top rank after FitSearch (Figure 3A and 3B).

In the second literature-based case study, we tested whether the new method could be applied to measure the similarity between a chemical-genetic profile and a genetic-interaction profile. In principle, deletion of a gene that encodes the target of an inhibitory compound should cause cellular effects that are similar to inhibition of the target by a drug treatment. This proof-of-principle experiment was successfully performed [19]. Our FitSearch is a generalization of this type of approach. We showed that the target protein of clotrimazole, ERG 11 was detected using chemical- and genetic-genetic profiles generated in different platforms (Figure 3C).

FitSearch benefits researchers performing small-scale experiments using yeast deletion collection because in these studies, only one or two chemical-genetic profiles are generated by colony- or OD-based platforms. This makes it difficult to perform data mining from public resources, as these data are typically generated on the chip-based platforms. An example is a recent study (manuscript in preparation) that generated yeast fitness profiles to 2,4-DAPG, an antibiotic with a poorly understood mode-of-action. Through similarity searching of FitRankDB using the web-frontend, FitSearchp (Table 2), we found three similar compounds; menthol, sodium azide and $\mathrm{H}_{2} \mathrm{O}_{2}$ in the top rank (Figure 3D). Menthol is known to cause a perturbation in the lipid fraction of the membrane, altered membrane permeability and consequential leakage of intracellular materials [23]. Sodium azide has been known as a rapid and reversible inhibitor of the cytochrome c oxidase-respiratory chain complex $\mathrm{IV}$, through enhanced cytochrome c holoenzyme dissociation [24]. Membrane associated protein kinase $\mathrm{C}$ activity can also be altered by sodium azide [24]. Marino et al. [25] reported that sodium azide increases intracellular calcium in mammalian systems, causing azide neurotoxicity. Hydrogen peroxide $\left(\mathrm{H}_{2} \mathrm{O}_{2}\right)$ can damage proteins, lipids, and DNA. The primary source of reactive oxygen species such as $\mathrm{H}_{2} \mathrm{O}_{2}$ is free-leakage of electrons, which is generated by the mitochondrial respiratory system. Based on the modes-of-action of these three similar drugs, it was suggested that mechanism of action of 2,4DAPG may involve disturbing cell membrane permeability, triggering of a reactive oxygen burst, and interruption of cell homeostasis. 


\section{Conclusions}

Yeast deletion collections have been successfully used to infer mode-of-actions of drugs, in particular by profiling chip-based chemical-genetic and genetic-genetic interactions on a genome-wide scale. For optimal use of public resources, we designed FitRankDB as a general repository of fitness profiles, and developed FitSearch as a new similarity measure between such profiles. We showed that our new repository and algorithm are beneficial to researchers who are attempting to obtain hypothesis regarding the unknown modes-of-action of a bioactive compound through a small-scale experiment with yeast deletion collections from different platforms, specifically non-chip based platforms.

\section{Additional material}

Additional file 1: Summary of FitSeach results of Clotrimazole, Latrunculin B, Benomyl, Cisplatin, and 2,4-DAPG.

\section{Acknowledgements}

We thank all members of the Bioinformatics and Computational Biology Laboratory at KAIST for helpful discussions. This work was supported by the National Research Foundation of Korea (NRF) grant funded by the Korean Government, the Ministry of Education, Science \& Technology (MEST) [20090086964].

\section{Author details}

'Department of Bio and Brain Engineering, Korea Advanced Institute of Science and Technology, 291 Daehak-ro, Yuseong-Gu, Daejeon, 305-701, Republic of Korea. ${ }^{2}$ Bioinformatics Lab, Healthcare group, SK telecom, 9-1, Sunae-dong, Pundang-gu, Sungnam-si, Kyunggi-do, 463-784, Republic of Korea. ${ }^{3}$ Department of Biological Science, Seoul National University, 599 Gwanakro, Gwanak-gu, Seoul, 151-747, Republic of Korea. ${ }^{4}$ Department of Applied Biology, Gyeongsang National University, 501 Jinju-daero, Jinju, Gyeongnam, 660-701, Republic of Korea. ${ }^{5}$ USDA-ARS, Root Disease and Biological Control Research Unit, 367 Johnson Hall, Washington State University, Pullman, Washington 99164-6430, USA.

\section{Authors' contributions}

$\mathrm{ML}, \mathrm{SH}$ and DK conceived of the study. SH and $\mathrm{HC}$ designed an algorithm and implemented it. ML maintains the web service. YK and DW performed an experimental case study. ML, SH, YK and HC analyzed data. All authors wrote, read and approved the final manuscript.

\section{Declarations}

The publication costs for this article were funded by the Korean Government, the Ministry of Education, Science \& Technology (MEST) [20090086964].

This article has been published as part of BMC Genomics Volume 14 Supplement 1, 2013: Selected articles from the Eleventh Asia Pacific Bioinformatics Conference (APBC 2013): Genomics. The full contents of the supplement are available online at http://www.biomedcentral.com/ bmcgenomics/supplements/14/S1.

\section{Competing interests}

The authors declare that there are no competing interests.

Published: 21 January 2013

\section{References}

1. Winzeler EA, Shoemaker DD, Astromoff A, Liang H, Anderson K, Andre B, Bangham R, Benito R, Boeke JD, Bussey $H$, et al: Functional characterization of the $S$. cerevisiae genome by gene deletion and parallel analysis. Science 1999, 285(5429):901-906.

2. Kim DU, Hayles J, Kim D, Wood V, Park HO, Won M, Yoo HS, Duhig T, Nam M, Palmer G, et al: Analysis of a genome-wide set of gene deletions in the fission yeast Schizosaccharomyces pombe. Nat Biotechnol 2010, 28(6):617-623.

3. Giaever G, Chu AM, Ni L, Connelly C, Riles L, Veronneau S, Dow S, LucauDanila A, Anderson K, Andre B, et al: Functional profiling of the Saccharomyces cerevisiae genome. Nature 2002, 418(6896):387-391.

4. Lum PY, Armour CD, Stepaniants SB, Cavet G, Wolf MK, Butler JS, Hinshaw JC, Garnier P, Prestwich GD, Leonardson A, et al: Discovering modes of action for therapeutic compounds using a genome-wide screen of yeast heterozygotes. Cell 2004, 116(1):121-137.

5. Giaever G, Flaherty P, Kumm J, Proctor M, Nislow C, Jaramillo DF, Chu AM, Jordan Ml, Arkin AP, Davis RW: Chemogenomic profiling: identifying the functional interactions of small molecules in yeast 2004.

6. Han S, Kim D: Inference of protein complex activities from chemicalgenetic profile and its applications: predicting drug-target pathways. PLoS Comput Biol 2008, 4(8):e1000162.

7. Parsons AB, Lopez A, Givoni IE, Williams DE, Gray CA, Porter J, Chua G, Sopko R, Brost RL, Ho CH, et al: Exploring the mode-of-action of bioactive compounds by chemical-genetic profiling in yeast. Cell 2006, 126(3):611-625.

8. Hillenmeyer ME, Fung E, Wildenhain J, Pierce SE, Hoon S, Lee W, Proctor M, St Onge RP, Tyers $M$, Koller $D$, et al: The chemical genomic portrait of yeast: uncovering a phenotype for all genes. Science 2008, 320(5874):362-365.

9. Pierce SE, Davis RW, Nislow C, Giaever G: Genome-wide analysis of barcoded Saccharomyces cerevisiae gene-deletion mutants in pooled cultures. Nat Protoc 2007, 2(11):2958-2974.

10. Xie MW, Jin F, Hwang H, Hwang S, Anand V, Duncan MC, Huang J: Insights into TOR function and rapamycin response: chemical genomic profiling by using a high-density cell array method. Proc Natl Acad Sci USA 2005, 102(20):7215-7220

11. Baek ST, Kim DU, Han S, Woo IS, Nam M, Kim L, Heo KS, Lee H, Hwang HR, Choi SJ, et al: Genome-wide drug-induced haploinsufficient screening of fission yeast for identification of hydrazinocurcumin targets. J Microbiol Biotechnol 2008, 18(2):263-269.

12. Breslow DK, Cameron DM, Collins SR, Schuldiner M, Stewart-Ornstein J, Newman HW, Braun S, Madhani HD, Krogan NJ, Weissman JS: A comprehensive strategy enabling high-resolution functional analysis of the yeast genome. Nat Methods 2008, 5(8):711-718.

13. Collins SR, Schuldiner M, Krogan NJ, Weissman JS: A strategy for extracting and analyzing large-scale quantitative epistatic interaction data. Genome Biol 2006, 7(7):R63.

14. Fiedler D, Braberg H, Mehta M, Chechik G, Cagney G, Mukherjee P, Silva AC, Shales M, Collins SR, van Wageningen $S$, et al: Functional organization of the S. cerevisiae phosphorylation network. Cell 2009, 136(5):952-963.

15. Schuldiner M, Collins SR, Thompson NJ, Denic V, Bhamidipati A, Punna T, Ihmels J, Andrews B, Boone C, Greenblatt JF, et al: Exploration of the function and organization of the yeast early secretory pathway through an epistatic miniarray profile. Cell 2005, 123(3):507-519.

16. Wilmes $G M$, Bergkessel M, Bandyopadhyay $S$, Shales M, Braberg $H$, Cagney G, Collins SR, Whitworth GB, Kress TL, Weissman JS, et al: A genetic interaction map of RNA-processing factors reveals links between Sem1/ Dss1-containing complexes and mRNA export and splicing. Mol Cell 2008, 32(5):735-746.

17. Fury W, Batliwalla F, Gregersen PK, Li W: Overlapping probabilities of top ranking gene lists, hypergeometric distribution, and stringency of gene selection criterion. Conf Proc IEEE Eng Med Biol Soc 2006, 1:5531-5534

18. Flaherty P, Giaever G, Kumm J, Jordan Ml, Arkin AP: A latent variable model for chemogenomic profiling. Bioinformatics 2005, 21(15):3286-3293.

19. Parsons AB, Brost RL, Ding H, Li Z, Zhang C, Sheikh B, Brown GW, Kane PM, Hughes TR, Boone C: Integration of chemical-genetic and genetic interaction data links bioactive compounds to cellular target pathways. Nat Biotechnol 2004, 22(1):62-69.

20. Stark C, Breitkreutz BJ, Reguly T, Boucher L, Breitkreutz A, Tyers M: BioGRID: a general repository for interaction datasets. Nucleic Acids Res 2006, 34(Database):D535-539.

21. Tong AH, Evangelista $M$, Parsons $A B, X u H$, Bader GD, Page N, Robinson M,

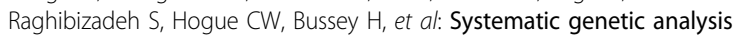


with ordered arrays of yeast deletion mutants. Science 2001, 294(5550):2364-2368.

22. Costanzo M, Baryshnikova A, Bellay J, Kim Y, Spear ED, Sevier CS, Ding H, Koh JL, Toufighi K, Mostafavi S, et al: The genetic landscape of a cell. Science 2010, 327(5964):425-431.

23. Trombetta D, Castelli F, Sarpietro MG, Venuti V, Cristani M, Daniele C, Saija A, Mazzanti G, Bisignano G: Mechanisms of antibacterial action of three monoterpenes. Antimicrob Agents Chemother 2005, 49(6):2474-2478.

24. Leary SC, Hill BC, Lyons CN, Carlson CG, Michaud D, Kraft CS, Ko K, Glerum DM, Moyes CD: Chronic treatment with azide in situ leads to an irreversible loss of cytochrome $c$ oxidase activity via holoenzyme dissociation. J Biol Chem 2002, 277(13):11321-11328.

25. Marino S, Marani L, Nazzaro C, Beani L, Siniscalchi A: Mechanisms of sodium azide-induced changes in intracellular calcium concentration in rat primary cortical neurons. Neurotoxicology 2007, 28(3):622-629.

26. Cherry JM, Hong EL, Amundsen C, Balakrishnan R, Binkley G, Chan ET, Christie KR, Costanzo MC, Dwight SS, Engel SR, et al: Saccharomyces Genome Database: the genomics resource of budding yeast. Nucleic Acids Res 2012, 40(Database):D700-705.

27. Navarro $P$, Durrens $P$, Aigle M: Protein-protein interaction between the RVS161 and RVS167 gene products of Saccharomyces cerevisiae. Biochimica et biophysica acta 1997, 1343(2):187-192.

28. Palancade B, Liu X, Garcia-Rubio M, Aguilera A, Zhao X, Doye V: Nucleoporins prevent DNA damage accumulation by modulating Ulp1dependent sumoylation processes. Molecular biology of the cell 2007, 18(8):2912-2923.

29. Wilmes GM, Archambault V, Austin RJ, Jacobson MD, Bell SP, Cross FR: Interaction of the S-phase cyclin Clb5 with an "RXL" docking sequence in the initiator protein Orc6 provides an origin-localized replication control switch. Genes \& development 2004, 18(9):981-991.

doi:10.1186/1471-2164-14-S1-S6

Cite this article as: Lee et al:: FitSearch: a robust way to interpret a yeast fitness profile in terms of drug's mode-of-action. BMC Genomics 2013 14(Suppl 1):S6.

\section{Submit your next manuscript to BioMed Central and take full advantage of:}

- Convenient online submission

- Thorough peer review

- No space constraints or color figure charges

- Immediate publication on acceptance

- Inclusion in PubMed, CAS, Scopus and Google Scholar

- Research which is freely available for redistribution

Submit your manuscript at www.biomedcentral.com/submit 